\title{
INVESTIGACIÓN
}

Recibido: 21/03/2019 --- Aceptado: 08/04/2019 --- Publicado: 15/09/2019

\section{MARKETING SENSORIAL: EL CONCEPTO, SUS TÉCNICAS Y SU APLICACIÓN EN EL PUNTO DE VENTA}

\section{Sensory marketing: the concept, its techniques and its application at the point of sale}

(1D) 18 Gloria Jiménez-Marín1: Universidad de Sevilla. España. gloria_jimenez@us.es

(D) $R^{6}$ Elena Bellido-Pérez: Universidad de Sevilla. España. ebellido@us.es

Ángela López-Cortés: Universidad de Sevilla. España. angelalopezc@us.es

\section{RESUMEN}

El marketing sensorial comprende una serie de decisiones estratégicas dirigidas a la estimulación de los sentidos en el público. Cuando estas decisiones se toman sobre el propio punto de venta, el público logra una experiencia completa que le acerca más aún al producto frente al que se sitúa, fomentando de este modo su compra final. Por ello, los establecimientos que emplean de manera adecuada este tipo de técnicas consiguen consolidar una experiencia de compra altamente satisfactoria apuntando al nivel subconsciente de los sentidos. No obstante, se deben tener en cuenta una serie de consideraciones a la hora de implementarlas para conseguir los objetivos deseados, teniendo presente los avances en el ámbito de la neurociencia. En el presente texto se realiza una exploración en el concepto de marketing sensorial, señalando sus distintas tipologías y posibilidades de aplicación. Se describe, en primer lugar, el potencial de los cinco sentidos como vías a través de las cuales dejar un recuerdo en el consumidor. Luego, se especifican las técnicas de medición y las pautas de implantación del marketing sensorial $\mathrm{y}$, finalmente, se detalla el proceso de compra que sigue el cliente en el establecimiento, indicando cómo podría estimularse la compra final con una estrategia que se dirigiese a los sentidos del consumidor.

\footnotetext{
${ }^{1}$ Gloria Jiménez Marín: Profesora Titular de la Universidad de Sevilla. Especialista en merchandising y autora los libros Merchandasing y retail. Comunicación en el Punto de Venta (Advook, 2016) y La gestión profesional del merchandising (UOC, 2017).
} 
Jiménez-Marín, G.; Bellido-Pérez, E. y López-Cortés, A. Marketing sensorial: el concepto, sus técnicas y su aplicación en el punto de venta.

PALABRAS CLAVE: marketing sensorial - neuromarketing - punto de venta merchandising - comunicación - marca - publicidad.

\begin{abstract}
Sensory marketing contains a set of strategic decisions focused on the sense's stimulation in the public. When these decisions are taken on the point of sale, the public accomplish a full experience that brings them close to the product that they are evaluating, motivating in this way the final purchase. Hence, the establishments that apply in an appropriate way this kind of techniques strengthen a highly satisfactory purchase experience, focusing on the subconscious level related to the sense. Nevertheless, a few considerations need to be taken into account when applying these techniques to get the desired objectives, being aware of the neuroscience advances. In this text, an exploration of sensory marketing concept is carried out, pointing out its various typologies and application possibilities. Firstly, the potential of the five senses as means of leaving a memory in the consumer is described. After that, measuring techniques and implementation guidelines are specified, and finally, the customer's buying process in the establishment is detailed, indicating how the final purchase could be stimulated with a senses-focused strategy.
\end{abstract}

KEY WORDS: sensory marketing - neuromarketing - point of sale - merchandising communication - branding - advertising.

\title{
MARKETING SENSORIAL: O CONCEITO, SUAS TECNICAS E SUA APLICAÇÃO NO PONTO DE VENDA
}

\section{RESUME}

O marketing sensorial compreende uma series de decisões estratégicas dirigidas a estimulação dos sentidos no público. Coando estas decisões tomadas sobre o próprio ponto de venda, o público logra uma experiência completa que o aproxima ainda mais ao produto fomentando deste modo sua compra final. Por isso, os estabelecimentos que empregam de maneira adequada este tipo de técnicas conseguem consolidar uma experiência de compra altamente satisfatória apontando ao nível subconsciente dos sentidos. Não obstante, se devem ter em conta uma serie de considerações na hora de implementa-las para conseguir os objetivos desejados, tendo presente os avances no âmbito da neurociência. No presente texto se realiza uma exploração no conceito de marketing sensorial, assinalando suas distintas tipologias e possibilidades de aplicação. Em primeiro lugar, se descreve o potencial dos cinco sentidos como vias através das quais deixar uma lembrança no consumidor. Logo, especificam as técnicas de medição e as pautas de implantação do marketing sensorial e, finalmente, se detalha o processo de compra que segue o cliente no estabelecimento indicando como poderia estimular a compra final com uma estratégia que se dirija aos sentidos do consumidor. 
Jiménez-Marín, G.; Bellido-Pérez, E. y López-Cortés, A. Marketing sensorial: el concepto, sus técnicas y su aplicación en el punto de venta.

PALAVRAS CHAVE: marketing sensorial - neuromarketing - ponto de venda merchandising - comunicação - marca - publicidade.

\section{Como citar el artículo:}

Jiménez-Marín, G.; Bellido-Pérez, E. y López-Cortés, A. (2019). Marketing sensorial: el concepto, sus técnicas y su aplicación en el punto de venta. [Sensory marketing: the concept, its techniques and its application at the point of sale]. Vivat Academia. Revista de Comunicación, 148, 121-147.

DOI: http://doi.org/10.15178/va.2019.148.121-147

Recuperado de http://www.vivatacademia.net/index.php/vivat/article/view/1177

\section{INTRODUCCIÓN}

\subsection{El marketing sensorial y sus tipologías}

El marketing sensorial, también denominado marketing de los sentidos, se puede entender de manera general, tal y como lo describe Krishna, como el marketing que se dirige a los sentidos de los consumidores y afecta a su comportamiento (2010, p. 2). Esto implica estudiar la sensación y percepción como vías que modifican el comportamiento del consumidor (Krishna, 2012, p. 333).

De manera más específica, el marketing sensorial en el punto de venta lo entenderemos como aquel que se dirige a los cinco sentidos buscando la estimulación de estos para intentar crear un ambiente agradable, de forma que el cliente incremente el tiempo de compra en el establecimiento. Este concepto se engloba en la ciencia denominada neuromarketing, encargada de estudiar los procesos mentales de los consumidores relacionados con la percepción, la memoria, el aprendizaje, la emoción y la razón, partiendo de la idea de que tras una respuesta consciente en el consumidor se hallan motivos profundos (Manzano et al., 2012, p. 72). Autores como Stanton, SinnottArmstrong y Huettel lo definen como "el uso de técnicas de investigación psicológicas y de neurociencia para ganar la afectividad en el comportamiento del consumidor, en sus preferencias y en su toma de decisiones" (2017, p. 800., traducción propia). Así, el neuromarketing "trata de explicar los factores que influyen y afectan a sus pensamientos, sentimientos, motivaciones, necesidades y deseos, para entender en última instancia qué define su comportamiento de compra" (Manzano et al., 2012, p. 72).

El marketing sensorial ha adquirido una importancia determinante en los últimos años en muchos sectores, puesto que es la mejor forma de conectar la marca o producto con el estilo de vida de nuestros clientes, agregando valor a su entorno, apelando a sus sentimientos, a sus sentidos y a la razón. De este modo, en palabras de Lindstrom (2012, p. 4), se trata de "lograr un compromiso emocional con el consumidor para que recuerde 
Jiménez-Marín, G.; Bellido-Pérez, E. y López-Cortés, A. Marketing sensorial: el concepto, sus técnicas y su aplicación en el punto de venta.

la marca, y para permanecer en su memoria debe tocar la fibra de sus sentimientos; por eso hay que crear una historia con la cual pueda identificarse y comprometerse". Por consiguiente, el cliente ya no elige un producto o servicio solo por la relación costebeneficio, sino por la vivencia que ofrece dicho producto y servicio, o por la percepción que pueda tener de este. En este punto, es importante destacar que aunque hay comportamientos que son manejados por la cultura en el subconsciente, otros, en cambio, son culturales.

Para que la empresa tenga éxito mediante el uso del marketing de los sentidos es necesario que la estrategia esté integrada. Es decir, utilizar técnicas cotidianas en las que todos los sentidos influyan de alguna manera para intentar vender más (Valenti y Riviere, 2008). No es suficiente que se vea o se hable del producto; es necesario que sea experimentado y se asocie con un estilo de vida determinado. La integración sensorial es de suma importancia ya que, de lo contrario, los efectos en los consumidores pueden ser contraproducentes, y por lo tanto traerá como consecuencia confusiones y, lejos de mejorar la percepción, traería resultados negativos: se daría, con ello, un antimarketing.

Por tanto, se pueden definir, en primer lugar, los sentidos gestionados por el marketing sensorial, paso previo para plantear una estrategia coherente con la imagen de marca. Así, se pueden distinguir el marketing olfativo, táctil, sonoro y gustativo.

Respecto al marketing olfativo, es altamente significativo apuntar que los seres humanos recordamos el 35\% de lo que olemos (Díaz, 2012). Además de ello, la nariz es capaz de distinguir más de un trillón de estímulos olfativos, hecho comprobado por el equipo de Andreas Keller (Bushdid et al., 2014), siendo el olfato uno de los sentidos más sensibles y emocionales, con una gran capacidad para asociar determinados olores con situaciones concretas. De hecho, estudios relacionados con el neuromarketing afirman que el $75 \%$ de nuestras emociones están relacionadas con los olores (Jiménez-Marín, 2016), de ahí que los olores sean susceptibles de impactar sobre el comportamiento de compra de los consumidores. En este sentido, diversas investigaciones del SOSI (Sense of Smell Institute) indican que mientras que los humanos tan sólo son capaces de recordar al cabo de tres meses el 50\% de las cosas que han visto, en el caso de los olores, ese porcentaje puede aumentar hasta un 65\% tras un año (Mukherjee, 2015, p. 1294).

El tacto, por otro lado, favorece la identidad de marca, ya que supone una doble interacción de los clientes con el producto. "Pasamos nuestras manos por el producto para acceder a sus propiedades, leerlo de forma táctil y generar una percepción o un sentimiento" (Manzano et al., 2012, p. 181). El tacto puede ser considerado como un medio para controlar la "inconsciencia de los consumidores, sus percepciones, sensaciones y preferencias" (Lobato, 2005, p. 11). El marketing táctil puede ser utilizado en diferentes ocasiones de acercamiento entre los consumidores y los productos (marcas). Nos referimos a las propias cualidades de los productos (textura, tamaño, 
Jiménez-Marín, G.; Bellido-Pérez, E. y López-Cortés, A. Marketing sensorial: el concepto, sus técnicas y su aplicación en el punto de venta.

materiales, etc.) y también al propio punto de venta. De hecho, el sentido del tacto dentro del establecimiento cobra una importancia vital, por ejemplo, en productos textiles, puesto que ofrece la posibilidad de interactuar con las prendas, diferenciándose así de la venta online (Jiménez-Marín, 2017).

El oído, por su parte, supone el segundo sentido más utilizado por detrás del sentido de la vista, y sirve para despertar emociones y sentimientos que influyen en la relación de los consumidores con las marcas. A pesar de que estudios especializados afirman que las personas recordamos el $2 \%$ de los sonidos que escuchamos (Díaz, 2012), lo cierto es que este tipo de marketing usa un porcentaje sustancialmente inferior al de otros sentidos. No obstante, la música es uno de los elementos claves para la creación de la imagen del punto de venta y la identidad de la marca, ya que puede ser utilizada para ayudar a establecer una imagen de marca en la mente de los usuarios, además de para influenciar sus hábitos de compra en el propio punto de venta. Relacionado con ello, el ritmo la música como ambiente de compra ayuda en los objetivos del establecimiento con unas pautas bien diferenciadas (Jiménez-Marín, 2016). La música lenta, por un lado, ayuda a la relajación y, por tanto, a realizar compras de manera más sosegada, incrementando las posibilidades de compra. La música rápida, por su parte, ayuda a la acción y, por consiguiente, a realizar compras de manera más eficiente, no aumentando las posibilidades de compra pero sí ayudando a evitar cuellos de botella y, por tanto, aumentando la satisfacción de compra del cliente.

Por último, en relación al marketing gustativo, el sentido del gusto está relacionado con los estados emocionales, por lo que puede contribuir a cambios de actitud y de percepción de marca. Además, para que el producto llegue a estar en contacto con este sentido, debe pasar por el resto de los sentidos, ya que éstos actúan como filtros (Manzano et al., 2012, p. 159). Es el sentido más complejo, ya que "ningún otro exige el complemento de la totalidad del resto de los sentidos para cumplir su función y enviar informes sensoriales completos al cerebro"; por tanto, la integración del gusto como sentido genera "emociones más ricas y completas, que logran incluso trasladarnos directamente en el tiempo por medio de la memoria, y recordar sabores lejanos" (Manzano et al., 2012, p. 159). Así, el gusto suele ser uno de los principales reclamos del segmento de la hostelería y los alimentos: desde bares o restaurantes (que ofrecen alimentos con un sabor reconocible) a supermercados (que intentan atraer la atención de los potenciales compradores con alimentos de prueba) o incluso marcas de pequeños electrodomésticos.

El marketing sensorial, en definitiva, y tal y como expresa Castanyol (2014) intenta establecer nuevos vínculos con los públicos potenciales mediante los sentidos. El objetivo es despertar emociones que tengan mucha más fuerza que nuestra parte racional a la hora de escoger un producto o un servicio. En este sentido, autores como Haase y Wiedmann han desarrollado una escala de percepciones y sensaciones que 
Jiménez-Marín, G.; Bellido-Pérez, E. y López-Cortés, A. Marketing sensorial: el concepto, sus técnicas y su aplicación en el punto de venta.

puede experimentar el consumidor a través del marketing sensorial: se trata del SPI (sensory perception item), que identifica 20 adjetivos a potenciar (2018). El SPI se materializa en una tabla que asocia cuatro adjetivos a cada sentido (como por ejemplo "comfortable", "handy", "soothing" y "well-shaped" para el sentido háptico), elaborada por los autores tras una revisión de la literatura, una entrevista a expertos y un estudio de campo realizado en un punto de venta. En esta investigación, Haase y Wiedmann determinan que la actitud frente al producto, la recomendación boca-oreja y la intención de compra guardan una relación significativa con el SPI, por lo que puede usarse para determinar los efectos del marketing sensorial (2018, p. 11).

\subsection{Algunos conceptos clave en el marketing sensorial}

A la hora de tomar decisiones de compra, se hace necesario aclarar dos conceptos clave como son los marcadores somáticos o la atención implícita. Así, partimos de que los consumidores pueden usar mecanismos únicamente emocionales e inconscientes, construidos en torno los denominados marcadores somáticos. De hecho, y partiendo de las dos grandes vías de comunicación y persuasión, la racional y la emocional que citan Ramos et al. (2007) por las que "la vía racional pretende convencer ofreciendo razones o argumentos, mientras que la emotiva pretende atraer al receptor desde la fascinación" (Ferrés, 1996, p. 70), podríamos afirmar que el porcentaje de decisiones de compra que se toman en base a cuestiones emocionales supera a las racionales (Ramos et al., 2007).

En este sentido, Damasio (1994) ya hacía alusión a que emociones y razón no son extremos, sino tramos de una escala que ayudan a los compradores a tomar sus decisiones de compra. Esta teoría es fundamental a la hora de entender el comportamiento del consumidor, porque plantea la forma en la que las emociones se relacionan con la atención y la memoria, entre otros, influyendo, de este modo, en las reacciones y respuestas ante productos y servicios y ante estímulos publicitarios y de marketing.

Este autor denominó como 'marcadores somáticos' a los elementos (sensaciones y emociones) que impactan en la percepción, evaluación, decisiones y comportamiento de los consumidores a la hora de ejecutar una compra potencial. Es, básicamente, la existencia de un mecanismo emocional para tomar decisiones basado en el tradicional modelo de aprendizaje que ya planteado por el modelo AIDA (Sánchez, 1999). Concibe que las experiencias evocan ciertos sentimientos y, por tanto, determinadas reacciones a través del sistema nervioso automático. Estas sensaciones y emociones se almacenan como marcadores somáticos y tienes consecuencias en la toma de decisiones de los compradores. De esta forma se toman decisiones por medio de un mecanismo emocional, sin necesidad de pasar por un pensamiento racional (Santos, 2018). 
Jiménez-Marín, G.; Bellido-Pérez, E. y López-Cortés, A. Marketing sensorial: el concepto, sus técnicas y su aplicación en el punto de venta.

Asimismo, la atención en las decisiones de compra también juega un papel importante pues "la atención, junto con la memoria y la motivación suponen los tres dispositivos básicos del aprendizaje" (Llinás, 2003, p. 27). Y es aquí donde destacamos el concepto de atención implícita, que es, inconsciente e involuntaria. Y contraria a la atención explícita, que es consciente y se puede gestionar de forma cognitiva. La atención implícita se encuentra directamente relacionada con el denominado SARA: Sistema Activador Reticular Ascendente, que funciona como un primer filtro para los estímulos que entran en el cerebro del posible consumidor, filtrando, aproximadamente, el 95\% de la información (Serrano \& De Balanzó, 2010). Así, valora cada estímulo y sólo permite pasar aquello que le llama especialmente la atención y plantea como pertinente. Esto es, tal y como plantean Stanton et al. (2017): Colores, sonidos, olores o movimientos llamativos.

\subsection{De la Unique Selling Proposition a la Me Selling Proposition}

La publicidad en el futuro no estará basada en el consciente, sino en el subconsciente. En esta línea, según Lindstrom, "el $85 \%$ de las decisiones que tomamos a diario, depende de nuestro subconsciente" (2012, p. 10). Los consumidores, tal y como reflexiona Debans, "son seres sensoriales que necesitan relacionarse con el producto desde ángulos innovadores, participar de la búsqueda y develación de la marca y la esencia del mismo", puesto que "se experimenta el mundo a través de los sentidos y es a través de éstos que entra el conocimiento a nuestro cerebro" (2005).

Es por ello que las degustaciones de determinados productos alimentarios son cada vez más habituales en los puntos de venta, a la vez que en el diseño de packaging se trabaja con materiales y texturas que resulten agradables al tacto.

El marketing sensorial intenta ir más allá y romper la barrera de la publicidad audiovisual. Ahora se pretende reforzar los valores y las características diferenciales de determinados productos, servicios o incluso eventos, tal y como aportan Guerra y Gomes-Franco (2017). En este sentido, la decoración, la iluminación, las texturas de los materiales y la aromatización de espacios son elementos con los que se juega para crear experiencias únicas en determinadas tiendas, centros comerciales, aeropuertos y hoteles (De Garcillán, 2015). Ejemplos claros de ello son el olor a café al entrar en una cafetería, la música acorde con el estilo del consumidor en un establecimiento, la compra de productos con un tacto especial, etc. Podemos destacar el caso del iPad, que ha sido tremendamente exitoso por incluir nuevas sensaciones para el consumidor, principalmente el tacto. Así, las marcas que apelan a múltiples sentidos tendrán más éxito que las que se centran sólo en uno o dos (Haase y Wiedmann, 2018).

Gracias a la neurociencia combinada con el marketing, las marcas pueden seguir patrones cada vez más sensoriales. Por esta razón, ahora se está pasando de una 
Jiménez-Marín, G.; Bellido-Pérez, E. y López-Cortés, A. Marketing sensorial: el concepto, sus técnicas y su aplicación en el punto de venta.

estrategia Unique Selling Proposition (USP), enfocada en el producto, a una estrategia de Me Selling Proposition (MSP), enfocada directamente hacia el consumidor y muy en línea con el concepto que Roberts (2005) ya proponía sobre las lovemarks. Con la MSP se potencia el amor a una marca, producto o establecimiento; se potencia la afinidad personal con un bien, poniendo en primer lugar al sujeto, al yo, al ego, como eje vertebrador de cualquier estrategia, de cualquier campaña. Así, el consumidor se siente dueño de la marca, protegiéndola y desarrollándola (Rowley, 2004, p. 134). Lejos de concebir la estrategia de venta de producto en las características del mismo, pasamos a concebirla pensando en lo que más importa al consumidor: él mismo y su experiencia hedonista. Es ahí donde el target pasa de querer a amar las marcas. En ese sentido, la MSP trabaja para potenciar esa egoquerencia, y para ello recurre con frecuencia al marketing sensorial.

\section{OBJETIVOS}

El objetivo principal del presente texto es el estudio del marketing sensorial como fenómeno para conocer las posibilidades que ofrece en su aplicación en el punto de venta y detallar así el modo en que el público puede responder gracias a él. Así pues, mediante una revisión teórica, el objetivo principal se desglosa en tres:

1. Revisar el concepto de marketing sensorial a través de un recorrido por las características principales de los cinco sentidos y el modo de estimulación de los mismos.

2. Estudiar las posibilidades de implantación del marketing sensorial en el punto de venta.

3. Estudiar la manera en la que el marketing sensorial estimula los sentidos y las emociones del público.

\section{LOS SENTIDOS: CARACTERÍSTICAS RELEVANTES PARA EL MARKETING SENSORIAL}

En línea con las aportaciones de Hulten (2011) o Jiménez-Marín y Elías (2018) el gusto

y el olfato son los sentidos más primitivos: aportan una información limitada e imprecisa del mundo exterior y están completamente interconectados; en cierta forma saboreamos con la nariz y olemos con la boca. Nuestro olfato se ha convertido en un apoyo para otros órganos que evolutivamente fueron más necesarios para la supervivencia de la especie (Gómez, 2012). Ello mismo le ocurre al tacto. De este modo, tacto, gusto y olfato no permiten obtener información espacio temporal por sí mismos. Sin embargo, la visión y el oído permiten tener una información espacio temporal real y más óptima. En este sentido, el primordial es el sentido de la vista, ya que el oído humano tiene limitaciones en cuanto al espectro de frecuencias sonoras que es capaz de percibir. 
Jiménez-Marín, G.; Bellido-Pérez, E. y López-Cortés, A. Marketing sensorial: el concepto, sus técnicas y su aplicación en el punto de venta.

\subsection{El oído}

Es uno de los sentidos que más influyen en las decisiones de compra. La denominada contaminación auditiva (sonidos o ruidos no deseados, elevados o inadecuados) pueden afectar el estado de ánimo de los clientes, a nivel consciente como inconsciente, lo que puede afectar negativamente a las ventas; por el contrario, sonidos adecuados pueden ayudar a incrementar las ventas, según hace constar el Informe POPAI del año 2016 (Popai, 2016). En este sentido destacamos que el denominado music branding, marketing sonoro o sonotipos (Gustems, 2005) crean una auténtica base de predisposición para los potenciales compradores.

Y es que la audición es, sin duda, un requisito previo para que se establezca comunicación entre empresa y target. Por ello, desde el punto de vista del neuromarketing es muy importante la distinción entre oír (percibir los sonidos a través del oído) y escuchar (prestar atención a lo que se oye). La memoria auditiva es un proceso cerebral dinámico que codifica y almacena información relacionada con las experiencias. En este punto, nos podemos preguntar de qué depende ese almacenamiento de información sonora, o por qué a veces recordamos perfectamente unos sonidos y obviamos completamente otros. El estado emocional previo del oyente, la personalidad, así como los intereses del individuo influyen en parte, pero son ya muchas las investigaciones que están consiguiendo localizar el área del cerebro que se encarga de la memorización de las canciones. Así, tal y como apunta Braidot (2011), en un estudio realizado con una muestra sobre estímulos sonoros musicales, los investigadores propusieron a los participantes escuchar diferentes canciones, entre ellas, Satisfaction (Rolling Stones) y la música de la Pantera Rosa. Se observó que el grado de actividad cerebral variaba si la música era lírica o sólo instrumental, y que canciones como Satisfaction permanecían en la memoria por mucho tiempo.

Los sonidos, en general, producen cambios en algunos de los sistemas de neurotransmisión y en la predisposición del cliente para hacerse con un producto o para entrar en un establecimiento. Porque la música genera cambios neuroquímicos que tienen gran influencia en la conducta. Al escuchar música la actividad cerebral se pone en marcha. Incluso ya hay estudios que apuntan a que los distintos tipos de música activan diversas partes del cerebro, entre ellas, el lóbulo frontal y el temporal (relacionado con las emociones que hacen llorar, reír y despertar el placer sexual) (Popai, 2016). En este sentido, es digno de reseñar que los diferentes estilos de música influyen en los niveles de presión arterial, aumentándola o disminuyéndola, al igual que en los niveles de energía y en el proceso de secreción hormonal (Braidot, 2011). Así, la música clásica o la new age provocan estados de ánimos positivos, la música con pocas alteraciones de ritmo y tono genera estados de calma y placer, y la música con altas alteraciones de ritmo, tono y volumen provoca sensaciones de rabia o tristeza (Braidot, 2011).

Vivat Academia. Revista de Comunicación. 15 septiembre 2019/15 diciembre 2019, nº 148, 121-147 
Jiménez-Marín, G.; Bellido-Pérez, E. y López-Cortés, A. Marketing sensorial: el concepto, sus técnicas y su aplicación en el punto de venta.

\subsection{El gusto}

El gusto es el resultado de procesar los sabores a través de las casi 10.000 papilas gustativas que el ser humano tiene en la boca. Actúa por contacto de sustancias químicas solubles con el órgano lingual y es capaz de percibir un abanico muy amplio de sabores, texturas o temperaturas. Existen cuatro sensaciones sápidas primarias: ácido, dulce, amargo y salado 2 . Sin embargo, y siguiendo a Gómez y García (2012), una persona puede percibir cientos de sabores concretos diferentes, que son las combinaciones de las cuatro sensaciones por combinaciones múltiples. Las papilas sensibles a los sabores dulce y salado se concentran en la punta de la lengua, las sensibles al ácido ocupan los laterales quedando, la parte posterior de la lengua para los sabores amargos.

El sentido del sabor es un sentido con ciertas limitaciones a la hora de usarlo por sus propias características: no podemos degustar una camiseta o un zapato. Sin embargo, en aquellos productos susceptibles de ser saboreados, las degustaciones o muestras de prueba, dan muy buenos resultados incrementando los niveles de venta hasta en un $95 \%$ (Popai, 2016). Asimismo, las personas recordamos aproximadamente el 15\% de lo que probamos, por lo que se trata de un nivel de retención mucho mayor que el que ofrecen los otros sentidos. Por eso, el sentido del gusto es uno de los principales reclamos del sector de la hostelería y los alimentos.

El gusto es susceptible a ser influenciado por estímulos del exterior, como el nombre de la marca, la información de los compuestos del producto ${ }^{3}$, el embalaje o la publicidad (Allison y Uhl, 1964; Leclerc, Schmitt y Dubé, 1994; Lee, Frederick y Ariely, 2006; Levin y Gaeth, 1988; Wansink et al., 2000; Mukherjee, 2015; o Nenkov, et al., 2018). En esta línea, el sentido del gusto, además, puede trabajarse no solo a través del contacto directo del producto con el consumidor, sino también a través de la sugerencia, esto es, mediante una presentación visual y un texto adecuados. Para ello, el gusto debe combinarse armoniosamente con el resto de sentidos. En este sentido destaca la investigación de Krishna, Cian y Sokolova, quienes demostraron que cuando una pieza publicitaria sobre comida se refería al resto de sentidos además del gusto el producto se percibía como más sabroso (2016, p. 144).

La relación entre algunos sabores y sensaciones determinadas suele ser también materia de conocimiento popular, como la unión directa entre el chocolate y el placer,

\footnotetext{
2 No obstante, el profesor Kikunae Ikeda, de la Universidad Imperial de Tokio identificó un quinto sabor apreciable en los espárragos, tomates, queso y carne, y lo denominó umami, que significa "sabroso" (Manzano et al., 2012 p. 160).

${ }^{3}$ Los sabores de las bebidas están muy influenciados por su color. Los zumos de fruta llevan colorantes para potenciar la asociación con su sabor, y la gran mayoría de los refrescos de cola son marrones por un colorante. Sin embargo, existe una marca comercial en Perú, Inca Cola, mucho más natural que las grandes marcas comerciales, cuyo color, al no usar colorantes, es amarillo intenso y flúor.
} 
Jiménez-Marín, G.; Bellido-Pérez, E. y López-Cortés, A. Marketing sensorial: el concepto, sus técnicas y su aplicación en el punto de venta.

por ejemplo. Estas relaciones conocidas pueden ser usadas en otro tipo de productos no gastronómicos o establecimientos no relacionados directamente con la hostelería.

\subsection{El olfato}

Un olor provoca muchas emociones, y, además, nos encontramos ante el sentido que posee una conexión más directa con la memoria (Krishna, 2012). Sin embargo, el marketing olfativo es una herramienta muy nueva, pero que ha suscitado mucho interés en todo el mundo por la captación de varios aromas con la finalidad de dejar recuerdos en el consumidor final. Uno de los pioneros en aplicar el marketing olfativo fue Walt Disney, quien impregnó su parque de atracciones con aroma a palomitas recién hechas (con lo que consiguió un fuerte aumento de venta de palomitas en los quioscos y carritos ambulantes de Disneyland). De hecho, el marketing olfativo en los establecimientos alimenticios es clave. Investigaciones recientes han demostrado, por ejemplo, que una exposición prolongada a un olor de un alimento procesado en el establecimiento conduce a una compra menor de ese tipo de comida (Biswas y Szocs, 2019).

Tal y como afirman Gulas y Bloch (1995), Vroon (1999) o Bushdid et al. (2016), el olfato juega un papel importante en el reconocimiento de marca, al igual que el oído y la vista, ya que a través del cerebro se crea una vinculación física entre el sistema olfativo y el sistema límbico del cerebro, que puede producir un fuerte placer (Toller y Dodd, 2013). En el marketing olfativo entran en juego dos cualidades de los aromas para evaluar su uso: placer y congruencia. El placer recoge la experiencia intrínseca del olor, mientras que congruencia conecta el olor con el contexto: tienda, producto o marca.

Varios estudios, como los planteados por Gulas y Bloch (1995) o Gómez (2012) destacan las siguientes cualidades en los olores: la calidad afectiva del olor, el carácter estimulante y la intensidad. En cuanto al primero, el placer es el responsable de que los olores nos resulten agradables o desagradables. A su vez, la agradabilidad (o no) viene marcada por el tono, la esencia del aroma, la intensidad, el grado de concentración y la familiaridad. Por su parte, el carácter estimulante se refiere a si los olores evocan una respuesta fisiológica. Y la intensidad apunta a si el olor es fuerte o suave. Esto lo demostraban en un estudio reciente Bushdid et al. (2016).

Aprovechando las capacidades que ofrece el olfato, se está trabajando en la creación de odotipos ${ }^{4}$ eficaces capaces de transmitir aromas. Así, en línea con las aportaciones de Biswas \& Szocs (2019) el objetivo que genera todo este marketing de aromas es que se

\footnotetext{
${ }^{4}$ Un odotipo es el olor que identifica y caracteriza a un establecimiento o marca concretos, concebido para evocar y provocar ítems mentales a tener en cuenta para el marketing sensorial como son: Sensaciones, percepciones, emociones, sentimientos, pensamientos o actuaciones cada vez que sea percibido por el target.
} 
Jiménez-Marín, G.; Bellido-Pérez, E. y López-Cortés, A. Marketing sensorial: el concepto, sus técnicas y su aplicación en el punto de venta.

pueda lograr un contacto emocional con el consumidor para que recuerde la marca y se identifique con ella.

Si acudimos a los datos aportados por el estudio de Toller \& Dodd (2013) podemos afirmar que en todas las fragancias existen tres fases de percepción olfativa. En la base, las notas de fondo reafirman la identidad del perfume y otorgan el carácter del mismo. Es la base del perfume y dichas notas se perciben a partir de las dos horas. Suele relacionarse con colores oscuros y notas musicales graves. En la cima se hallan las notas de salida: es la primera impresión que se tiene del perfume cuando se pulveriza. La salida de una fragancia está formada por los componentes más volátiles. No suele durar más de quince minutos y comienza a percibirse a los 30 segundos de la aplicación de la fragancia. Esta primera percepción es muy importante en el momento de elegir una fragancia, ya que, de una manera casi instantánea, el perfume agrada o desagrada. Las notas de salida suele relacionarse con colores claros y notas musicales agudas. Y, en la zona central, se hallan las notas de corazón. Esta zona tiene los ingredientes principales de la fragancia y determina el espíritu real del perfume, constituyendo su personalidad. El aroma se aprecia minutos después de su aplicación y puede durar hasta cuatro horas. Suele relacionarse con colores intensos y notas musicales medias. Esto mismo viene refrendado en textos como los aportados por Jiménez-Marín y Elías (2018).

\subsection{El tacto}

El sentido háptico (del tacto) comprende la percepción de estímulos mecánicos (aplicación de una fuerza sobre una superficie) que incluyen contacto, presión y golpeo sobre la piel. Bajo la piel existen una serie de terminaciones nerviosas que hacen que los estímulos a través de la dermis sean interpretados de diversas maneras por el cerebro humano, ya que, en línea con las afirmaciones de Peck, Barger \& Webb (2013), cada terminación nerviosa supone una experiencia distinta susceptible de tener su reflejo en marketing. De este modo, y aunque el tacto es uno de los sentidos que menos se tiene en cuenta por su dificultad de implantación, también es cierto que juega un papel fundamental en las decisiones de compra: actúa como puente hacia la acción cuando un cliente está motivado para realizar la compra (Petit, Velasco \& Spence, 2019). Porque la percepción háptica se apoya en las fuerzas que se experimentan durante el contacto y puede servir para apreciar las calidades finales de un producto o para hacer que el potencial cliente interactúe con el propio producto. Ese contacto directo que mantiene el cliente con el producto conlleva también una potenciación del sentimiento de propiedad (Peck, Barger y Webb, 2013; Shu y Peck, 2011; Peck y Shu, 2009), lo cual está directamente relacionado con el concepto de Me Selling Proposition (MSP) que mencionamos anteriormente.

A pesar de su dificultad de implantación y su dependencia del tipo de producto y establecimiento, el sentido del tacto ha demostrado ser determinante a la hora de 
Jiménez-Marín, G.; Bellido-Pérez, E. y López-Cortés, A. Marketing sensorial: el concepto, sus técnicas y su aplicación en el punto de venta.

evaluar incluso experiencias online, donde los consumidores se han sentido más cercanos al producto a través de una pantalla táctil que utilizando el ratón (cfr. Petit, Velasco y Spence, 2019, p. 49).

\subsection{La vista}

La visión es una función cerebral que transforma la información impregnada en la retina en imágenes. Permite al ser humano interpretar la información recibida, conectándola con otros sistemas sensoriales como la memoria. Así, junto con otros sentidos como el oído, la vista puede trasladar al consumidor a contextos externos con los que relaciona el producto, llegando a posibilitar incluso juicios morales, tal y como han demostrado Nenkov et al. (2019).

La vista es el sentido que más vende. De hecho, la disciplina de visual merchandising surge para potenciar los elementos visibles del establecimiento comercial. En este sentido podemos afirmar que el $90 \%$ de la información que percibe nuestro cerebro es visual, siendo las características visuales, en un gran número de casos, las responsables de las ventas finales. De este modo, tal y como afirma Santos (2013), el color es el atributo principal del reconocimiento de una marca para el $80 \%$ de los consumidores siendo, en un $85 \%$ de los clientes, el detonante en la decisión de compra. Además, de la misma manera en la que algunos alimentos se asocian con sensaciones determinadas, $\mathrm{o}$ la música con estados de ánimo, existe un amplio abanico de significados que se atribuyen al color en la cultura occidental ${ }^{5}$ (el amarillo y la alegría, el rojo y la pasión, el verde y la naturaleza...), los cuales se pueden implantar en el producto y el establecimiento para suscitar ciertos sentimientos.

Las opciones que ofrece el hecho de dirigirse al sentido de la vista del consumidor son casi infinitas e, indudablemente, las más explotadas en el punto de venta en comparación con el resto de sentidos. La ambientación del establecimiento se percibe en un primer momento a través del sentido de la vista, para luego ir completándose mediante el oído, el olfato y el tacto. Así, trabajar con formas y colores en línea con la imagen de marca supone estudiar las maneras de emocionar o impactar al consumidor desde la visión. En este sentido, destacan las posibilidades que ofrece el uso del arte en el establecimiento, afectando al comportamiento de compra a través de la vista (Bellido-Pérez, 2017).

\section{APLICACIÓN DEL MARKETING SENSORIAL: BENEFICIOS, LÍMITES Y PAUTAS DE IMPLANTACIÓN}

Con el auge de las nuevas tecnologías, las posibilidades para hacer más efectivas las acciones de marketing en la empresa cada día son mayores, y por ello se busca

\footnotetext{
5 Se deben tener en cuenta las diferencias de significados en torno a un mismo color en diferentes países del mundo (cfr. Aslsm, 2006).
} 
Jiménez-Marín, G.; Bellido-Pérez, E. y López-Cortés, A. Marketing sensorial: el concepto, sus técnicas y su aplicación en el punto de venta.

constantemente la acción perfecta, esa que lleve a la empresa a un siguiente nivel. Martin Lindstrom (2012) apunta que los consumidores se han vuelto inmunes a los estímulos de la publicidad tradicional, y por eso es necesario utilizar la neurociencia para crear nuevas estrategias de marketing.

Gracias al conocimiento de los consumidores, las empresas pueden motivarlos a comprar determinados artículos a través de los estímulos sensoriales. No obstante, tal y como apunta Lindstrom, existen límites éticos (2012). Algunos de esos límites han sido revisados por Stanton, Sinnott-Armstrong y Huettel, quienes reflexionan sobre el miedo extendido a que el neuromarketing pueda predecir todos los comportamientos del consumidor, algo que no podría darse (2017, p. 803), o, más aún, el miedo a que el neuromarketing pueda influir en el comportamiento de compra (2017, pp. 803-804). Los mismos autores también apuntan los beneficios de esta ciencia, como la comprensión de las necesidades del consumidor, el tratamiento de la adicción o la perfección de campañas de salud pública (2017, p. 809).

En este sentido, la Dirección General de Tráfico (DGT), por ejemplo, con su campaña "Tú eliges" pretende situar al espectador en el rol del que sufre un accidente al volante por un despiste o una infracción. Así, mediante un equipo de realidad virtual, compuesto por unas gafas y unos auriculares, el usuario se pone en la piel de un conductor en tres escenas similares, pero con distinto desenlace, que se proyectan sucesivamente. "Impactante", "es muy real", "cuando te colocan la manta por encima se te ponen los pelos de punta" o "esta iniciativa pretende transmitir el dramatismo y la dureza de las gravísimas consecuencias que pueden tener unas mala prácticas al volante", son algunos de los comentarios que la propia organización afirma haber recibido como respuestas tras la exposición a esta campaña (Ramiro, 2017).

Conociendo, pues, la fuerza que el estímulo de los sentidos tiene sobre el consumidor, también pueden sucederse efectos contraproducentes. Tal y como ya aportasen autores como Hernández (2005), Gómez-Ramírez (2012) o, más recientemente, Nenkov et al. (2018), un simple cambio en el color o sabor de los alimentos, la aparición de un olor extraño o una textura distinta de la habitual, son variaciones de un producto que podrían ser detectadas por el consumidor y hacer que disminuyese su aceptación o que rechazase el producto. En este sentido, podemos afirmar que los consumidores son conscientes de que los productos poseen, además de sus características técnicas o intrínsecas, ciertas características sensoriales que suponen aspectos fundamentales de los mismos. De este modo, en los últimos años la calidad sensorial está teniendo una influencia creciente en el marketing de los productos, de manera que muchos alimentos y productos de cuidado personal son diferenciados por sus características sensoriales, y son estas las que en gran medida determinan si el producto es comprado de nuevo (Ferrada, 2013). Esto conlleva la inclusión de las características sensoriales en las especificaciones de producto con el objetivo de que la calidad sensorial sea mantenida 
Jiménez-Marín, G.; Bellido-Pérez, E. y López-Cortés, A. Marketing sensorial: el concepto, sus técnicas y su aplicación en el punto de venta.

en todos los productos por igual y a lo largo de toda la vida del producto, al igual que son mantenidos otros parámetros físico-químicos y nutricionales.

\section{1. Ítems mentales a tener en cuenta para el marketing sensorial}

Para hacer una correcta gestión de la concepción de la estrategia de marketing mediante marketing sensorial, se deberán identificar los cinco ítems mentales y cerebrales que existen desde antes de la compra y durante el consumo, para elegir el que más le convenga al objetivo del negocio (Jiménez-Marín, 2016):

1. Sensaciones o percepción. Se trata de satisfacer al consumidor por medio de los sentidos, creando experiencias a través de la vista, el oído, el tacto, el gusto y el olfato. Es importante definir muy bien cómo se va a generar ese impacto sensorial y debe diferenciarse claramente de los competidores para lograr esa motivación excepcional añadiendo valor a la marca, producto o servicio.

2. Emociones y sentimientos. En este caso se debe apelar a las emociones y sentimientos de los consumidores actuales y potenciales durante el proceso de venta y uso del producto. Pertenencia, alegría u orgullo son algunos de los sentimientos que enlazan de forma positiva y emocionalmente con las marcas, así que se debe conocer muy bien al comprador para escoger un camino que genere empatía a través de los estímulos adecuados.

3. Pensamientos. En este punto, se debe reforzar el pensamiento creativo y positivo hacia el producto, creando experiencias cognitivas que resuelvan problemas y atraigan a los clientes. Sorpresa, intriga, duda y provocación despertarán el interés y la creatividad por su utilización.

4. Acción-Actuaciones. Se centra en generar experiencias relacionadas con estilos de vida, comportamientos, acciones corporales e interpersonales que enriquezcan la vida del target, enseñándole diferentes alternativas para hacer las mismas acciones. Un claro ejemplo son los modelos, como las celebridades, deportistas o actores.

5. Relación. Generalmente, este último ítem contiene los módulos anteriormente mencionados como sensaciones, sentimientos, pensamientos y actuaciones. Las relaciones involucran experiencias grupales que generan una red donde se recrean los valores de la compañía ${ }^{6}$.

\subsection{Programa de control de calidad sensorial}

Para mantener la calidad sensorial necesaria para la consecución de los objetivos planteados es obligatoria la implantación de un programa de control de calidad desde el punto de vista sensorial. Este programa, en base al estudio de Hernández (2005), conlleva: (1) el establecimiento de especificaciones, (2) la selección de un método

${ }^{6}$ Para una información completa sobre esta idea, ver el trabajo de Fernández y Gordillo (2015).

Vivat Academia. Revista de Comunicación. 15 septiembre 2019/15 diciembre 2019, nº 148, 121-147 
Jiménez-Marín, G.; Bellido-Pérez, E. y López-Cortés, A. Marketing sensorial: el concepto, sus técnicas y su aplicación en el punto de venta.

sensorial de evaluación del cumplimiento de estas especificaciones, y (3) un programa de muestreo.

En cuanto a los recursos necesarios, se requiere, en primer lugar, a un personal cualificado responsable del análisis sensorial. Se trata de profesionales que seleccionen el tipo de prueba sensorial necesaria y que asesoren en el control de calidad sensorial de los productos. Seguidamente, se debe seleccionar el tipo de prueba sensorial más adecuada combinando criterios de obtención de la máxima información crítica y al menor coste económico. Luego, hay que contar con un espacio físico donde evaluar las muestras. Esta sala de catas debe ser cómoda y tranquila, libre de ruidos y olores y bien iluminada. Y, en último lugar, se reúne un equipo de medida a nivel sensorial (panel de catadores). Así, se debe seleccionar un grupo de personas con determinadas habilidades sensoriales y disponibilidad para la realización de los controles. Los catadores llegan a obtener una precisión u reproducibilidad válidas para la toma rápida y eficaz de decisiones en control de calidad. En un primer momento suelen estar influenciados por sus gustos y opiniones personales, pero el control de calidad se consigue gracias al entrenamiento, a la correcta elección de la prueba sensorial y a su puesta a punto en condiciones estandarizadas.

\subsection{Técnicas de medición de neuromarketing aplicadas al marketing sensorial}

Que el marketing comenzara a ir de la mano de la ciencia supuso un estudio en profundidad de la rama de la investigación de mercados que utiliza sistemas de medición biométrica. Esto es fundamental en cualquier control de calidad de técnicas de marketing sensorial correcto y objetivo. Muestra de su relevancia son las más de 200 empresas en todo el mundo dedicadas exclusivamente al desarrollo de estas técnicas para su aplicación posterior al marketing (Plassmann, Ramsoy y Milosavljevic, 2012). Dichas técnicas de medición en neuromarketing son las siguientes (Monge y Fernández, 2011; Stanton, Sinnott-Armstrong y Huettel, 2017):

- Electroencefalografía. Es una de las técnicas electrodiagnósticas de uso más extendido en la práctica médica y en las consultoras de neuromarketing. Consiste en el registro de la actividad eléctrica del cerebro mediante electrodos aplicados sobre el cuero cabelludo. Estos electrodos se colocan sobre las zonas correspondientes a las diferentes áreas del cerebro para así detectar y registrar patrones de actividad eléctrica y verificar la presencia de anomalías, disrupciones o efectos tras la aplicación de tácticas sensoriales. No se conforma con las declaraciones verbales de los sujetos, sino que pretende ir más allá, desentrañando el efecto del inconsciente y las emociones en la toma de decisiones. No obstante, la electroencefalografía "tiene una resolución espacial muy militada (según el número de electrodos, desde dos a varios cientos) y no ofrece datos de las partes internas del cerebro" (Monge y Fernández, 2011, p. 23).

- Imagen por resonancia magnética. Los estudios con imágenes por resonancia magnética (IRM) usan un gran imán y ondas de radio para observar órganos y 
Jiménez-Marín, G.; Bellido-Pérez, E. y López-Cortés, A. Marketing sensorial: el concepto, sus técnicas y su aplicación en el punto de venta.

estructuras que se encuentran en el interior del cuerpo. Los profesionales de la salud utilizan estas imágenes para diagnosticar una variedad de afecciones, desde rupturas de ligamentos hasta tumores. Las imágenes por resonancia magnética son muy útiles para examinar el cerebro y la médula espinal. En marketing sensorial se utilizan las resonancias magnéticas para, de nuevo, observar anomalías comportamentales de los individuos tras la aplicación de olores, sabores o sonidos, entre otros.

- Respuesta galvánica de la piel. Las técnicas de medición de la respuesta galvánica también se utilizan en neuromarketing como otro indicador más del estado del sujeto mientras es sometido a estímulos (normalmente publicitarios). Puesto que el incremento de conductividad de la piel representa una activación del sistema de "pelea o huye" del organismo, la conductancia de la piel es una excelente medida de activación/estimulación. No obstante, no ofrece información sobre la dirección o valencia de la emoción (si es positiva o negativa). Por lo tanto, normalmente se puede utilizar la respuesta galvánica para saber que existe una activación emocional pero son necesarias otras técnicas para determinar si se trata de deseo, miedo, ira...

- Eye-tracking. Es el proceso de evaluar correcta y objetivamente el punto donde se fija la mirada, o el movimiento del ojo en relación con la cabeza. Este proceso es utilizado en la investigación en los sistemas visuales, en psicología, en lingüística cognitiva y en diseño de productos. También en la elaboración de arquitectura web de tiendas online. Existen diversos sistemas para determinar el movimiento de los ojos. La variante más popular utiliza imágenes de vídeo a partir de las cuales se extrae la posición del ojo; otros métodos utilizan bobinas de búsqueda o están basados en electrooculogramas. Esta técnica necesita combinarse con otras como la electroencefalografía, "para saber exactamente qué está produciendo las reacciones cerebrales" (Monge y Fernández, 2011, p. 25).

- Ritmo cardíaco. El latido del corazón normalmente se mide en términos de tiempo entre latidos y se ha descubierto que las deceleraciones en el corto plazo suelen estar relacionadas con el incremento de la atención, a la vez que las aceleraciones a más largo plazo suelen corresponderse con el arousal ${ }^{7}$ emocional negativo (respuesta defensiva).

\section{EL CONSUMIDOR EN EL PUNTO DE VENTA: CÓMO POTENCIAR SUS SENTIDOS Y EMOCIONES}

\subsection{El proceso Atención-Interés-Deseo-Acción según pautas sensoriales}

El modelo de aprendizaje más extendido en el comportamiento de compra de un consumidor es un tema recurrente en eficacia publicitaria (Sánchez, 1999; Martí, 2012).

7 Es un término de base hipotética que describe los procesos que controlan la alerta, la vigilia y la activación cerebral frente a determinados estímulos.

Vivat Academia. Revista de Comunicación. 15 septiembre 2019/15 diciembre 2019, nº 148, 121-147 
Jiménez-Marín, G.; Bellido-Pérez, E. y López-Cortés, A. Marketing sensorial: el concepto, sus técnicas y su aplicación en el punto de venta.

Así, los modelos AIDA (Atención, Interés, Deseo, Acción) o AMBER (AtenciónMotivación-Brand Engagement-Respuesta) reflejan las distintas fases por las que pasa el ser humano a la hora de fijar su atención en un producto (o una marca) y llegar, finalmente, a comprarlo (o no). Con la variable atención se intenta llamar la atención del cliente potencial, captarla mediante alguno de los cinco sentidos para intentar conseguir estimular la siguiente variable, el interés por el producto marca. Se llama la atención, por ejemplo, atacando el sentido del olfato mediante el perfume de un establecimiento, mediante un escaparate visual y creativo o mediante una degustación. Si se consigue crear ese interés hacia el producto o marca se deberá aprovechar ese instante para explicar los beneficios que ofrece dicho producto o la marca. Así, se trata de activar en el cerebro la parte sensorial que permita que el potencial cliente quiera saber más. Tocar el producto, por ejemplo, puede ser un fiel reflejo de ese interés. Para pasar al siguiente ítem, el deseo, lo que se pretende es que el cliente desee el producto por encima de otro, sustitutivo o no, de la competencia o no. En el momento en que se obtiene la atención del potencial cliente es cuando se ha de persuadirle (o convencerle) de que el producto es "su producto". Si con estas tres variables se ha sido capaz de sentar las bases, el potencial cliente estará predispuesto para una acción de compra.

En este proceso psicológico individual inducido, las sensaciones, las percepciones y las emociones juegan un gran papel. Durante todo el recorrido, si se ha podido hacer que el cliente sienta, que se emocione y disfrute, se habrá conseguido una acción de venta inmediata.

\subsection{El consumidor frente a los estímulos sensoriales}

Se deberán distinguir, en primer lugar, los términos estímulo, sentido, sensación y percepción, tal y como ya indicase Castanyol (2014). Porque, diferenciemos: Un estímulo es cualquier tipo de energía a la que se puede responder; un sentido es una vía fisiológica particular por la que se puede responder a un tipo de energía específica; $y$, por su parte, la sensación es el sentimiento que se experimenta como respuesta a la información recibida a través de nuestros órganos sensoriales. Así, la percepción es la manera en que nuestro organismo organiza esos sentimientos para interpretarlos, es decir, es el reconocimiento de los objetos que proviene de combinar las sensaciones con la memoria de experiencias sensoriales anteriores.

En línea con esto, también se hace interesante conocer el concepto de umbral absoluto que, para Valenti \& Riviere (2008) es la intensidad más pequeña de un estímulo que puede percibirse. Así, estudios como los de Schiffman y Kanuk (2005), Hulten (2011) o Haase \& Wiedmann (2018) han demostrado que, en condiciones ideales, los sentidos humanos son capaces de percibir estímulos tan sutiles como los equivalentes estimados en la vida real. La expresión "en condiciones ideales" ya es significativa de que la sensibilidad de nuestros sentidos depende del nivel de fondo de la estimulación. Por su 
Jiménez-Marín, G.; Bellido-Pérez, E. y López-Cortés, A. Marketing sensorial: el concepto, sus técnicas y su aplicación en el punto de venta.

parte, el umbral diferencial es la diferencia más pequeña en intensidad requerida para que se pueda percibir diferencia entre dos estímulos. Esta relación entre el estímulo original y cualquier aumento o disminución es conocida como la ley de Weber (Schiffman y Kanuk, 2005), que fue el primero en advertir que cuanto mayor es el estímulo, mayor es el cambio para que pueda ser percibido.

En este punto, se puede hablar de las diferentes sensaciones y experiencias que puede tener un consumidor en los puntos de venta. Es decir, cómo reacciona el consumidor frente a los estímulos sensoriales a través de (Braidot, 2014):

- La adaptación. En ocasiones no se es consciente de ciertas variaciones en los estímulos debido a que se ha sufrido una adaptación a los mismos. La mente del consumidor se ha habituado a un nivel determinado de estimulación y ya no responde a ella de manera consciente. Adaptarse, por lo tanto, es disminuir los niveles de respuesta de los receptores sensoriales sometidos a continua estimulación.

- La atención. Al vivir rodeados de estímulos, una persona no puede percibir todos ellos a la vez. Pero si se fija la atención en alguno de ellos en concreto, este pasa al primer plano de su conciencia. En ocasiones, los estímulos son difíciles de ignorar, provocando así un efecto Stroop ${ }^{8}$.

- Los sistemas sensoriales. El sistema sensorial es parte del sistema nervioso y el responsable de procesar la información que se capta a través de los diversos sentidos. Está formado por receptores sensoriales y partes del cerebro involucradas en la recepción sensorial. Los principales sistemas sensoriales son los cinco sentidos, pero no son los únicos. Entran en juego otros sentidos tales como el vestibular, encargado de advertirnos si vamos hacia arriba o hacia abajo, el propioceptivo, que hace notar la posición de los miembros, el cenestésico, encargado de la tensión muscular y el equilibrio, o el interoceptivo, que da información sobre los órganos internos.

\section{CONCLUSIONES}

En definitiva, podemos aportar que, convertir el acto de compra en una experiencia agradable y provocar un recuerdo positivo y perdurable en el consumidor es posible gracias a los conceptos que se han detallado en el presente texto. Lo cierto es que todos los negocios intentan ganar clientes a través de la vista, del oído, etc., pero son pocos los que explotan o pueden explotar otras técnicas que si bien no reemplazan a otras, sí las pueden complementar y así crear en el subconsciente de los clientes un ambiente propicio al consumo. En esta línea podemos afirmar que, para tener éxito, el marketing

\footnotetext{
${ }^{8}$ El efecto Stroop es una interferencia semántica en el tiempo de procesamiento y reacción de una tarea concreta. El ejemplo más conocido es el de escribir una palabra como 'azul' en tinta de color rojo. Con esta situación se produce un retraso en el procesamiento del color de la palabra, lo que aumenta el tiempo de reacción ante el estímulo.
} 
Jiménez-Marín, G.; Bellido-Pérez, E. y López-Cortés, A. Marketing sensorial: el concepto, sus técnicas y su aplicación en el punto de venta.

debería abarcar todos los sentidos. El de la vista mediante el logo, el diseño de producto, el color, tipo de letra...; el del oído a través del tipo de música o bien el sonido del producto; el del gusto mediante el sabor del producto, obsequios o regalos comestibles; el del olor con una fragancia ambiental o con el propio aroma del producto; y el del tacto a través de la superficie y forma del producto, materiales de marketing o superficies ambientales.

En un mundo donde se reciben un promedio de 2,500 impactos publicitarios al día, de los cuales solo un 1\% logra su objetivo (Ferrada, 2013), es necesario incluir nuevas estrategias que permitan captar la atención y el corazón del comprador y que lo unan de forma constante a nuestra marca. Las empresas se apoyan estrategias de marketing mix tradicionales, basadas en la generación de reacciones en los clientes potenciales a través de la influencia de las $4 p^{\prime}$ s del marketing mix (product, price, place, promotion). Dado que esta combinación pretende captar la atención de los clientes, no es casualidad que las nuevas herramientas utilizadas para fidelizarlos estén orientadas hacia las emociones y los sentidos del que compra, pues el 95\% de las decisiones de compra se realizan de forma inconsciente y en la mayoría de los casos, las decisiones se toman desde la parte emocional y se justifican desde la parte racional (Cruz, 2012).

Así pues, fruto de nuestra experiencia, podemos aportar una serie de aspectos para cultivar la experiencia sensorial:

- A nivel visual, se puede trabajar sobre la ordenación, ambientación, jerarquización y la iluminación. La ordenación es esencial, pues un establecimiento desordenado vende un 5\% menos (Popai, 2016). La ambientación, por su parte, debe ser acorde con su posicionamiento: el cliente al entrar tiene que entender la tienda de manera inmediata haciendo un recorrido visual. El espacio, además, debe estar jerarquizado para que el cliente no se pierda por la tienda. Una jerarquía óptima es la que parte de la creación de escenarios, familias de productos, colores identificativos, y productos de más rotación a menos rotación. Respecto a iluminación, es de vital importancia tener muy bien iluminada la tienda, sobre todo si son tiendas profundas. El cliente no ha de sentir que está encerrado o en un lugar oscuro, ya que produce rechazo.

- A nivel sonoro, la música debe ser acorde al producto y al público objetivo, y la ambientación sonora debe ser modificada en función del momento del día y en función del día de la semana, así como diferente por estaciones.

- A nivel olfativo, es aconsejable disponer de un ambiente olorizado. Numerosos estudios avalan que la aplicación del marketing olfativo en comercios incrementa las ventas de forma considerable. Las reacciones provocadas por el uso de las técnicas de marketing olfativo tienen una explicación científica vinculada directamente con el funcionamiento de nuestro cerebro. Sin embargo, son pocos los establecimientos que usan esta técnica de manera consciente. 
Jiménez-Marín, G.; Bellido-Pérez, E. y López-Cortés, A. Marketing sensorial: el concepto, sus técnicas y su aplicación en el punto de venta.

- A nivel háptico, se recomienda que se permita tocar los productos y probar su uso, así como jugar con texturas y materiales en la arquitectura del establecimiento, lo cual puede ayudar a atraer la atención.

- En último lugar, a nivel gustativo, es aconsejable dar a probar los productos gastronómicos o culinarios y también potenciar sus olores. Aunque aquí nos refiramos al sentido del gusto lo cierto es que las papilas gustativas se activan también por el sentido del olfato.

En conclusión, al igual que Hultén proponía su "multi-sensory brand-experience concept" (2011), es decir, una marca interactiva en la que se integre la experiencia de todos los sentidos, y lo hacía a través del marketing sensorial, nosotros proponemos en la misma línea un establecimiento multi-sensorial, que haga de la experiencia de compra del cliente una experiencia única y vital. El potencial del marketing sensorial es indudable, ya sea aplicado a un producto concreto, a una marca o a un establecimiento. Las vías de contacto con las que el consumidor cuenta de antemano, esto es, los cinco sentidos, suponen caminos inmediatos para llegar a la emoción $y$, por consiguiente, mantenerse en el recuerdo del cliente. Una persona aprehende el mundo a través de los sentidos, y es en la combinación armoniosa y agradable de todos ellos donde se construyen experiencias inolvidables. Recrear ambientes o situaciones concretas, como un viaje a la India o la cena familiar de Navidad, es relativamente sencillo trabajando con ciertos olores, melodías, sabores, texturas y colores, facilitando así la asociación directa de un producto, marca o establecimiento a un ambiente determinado. Pero también se cuentan con infinitas combinaciones sensoriales para crear nuevas experiencias, para desarrollar nuevas concepciones que se iluminen en la mente del consumidor. Crear e implantar una estrategia de marketing sensorial adecuada es, en definitiva, la llave de acceso al recuerdo.

\section{REFERENCIAS}

Allison, R. I., y Uhl, K. P. (1964). Influence of beer brand identification on taste perception. Journal of Marketing Research, 1, 36-39.

Aslsm, M. (2006). Are you selling the right colour? A cross-cultural review of colour as a marketing cue. Journal of marketing communications, 12(1), 15-30.

Bellido-Pérez, E. (2017). Ambientación artística en los espacios comerciales, en JiménezMarín, G. (Ed.), La gestión profesional del merchandising (pp. 113-143). Barcelona: UOC.

Biswas, D. y Szocs, C. (2019). The Smell of Healthy Choices: Cross-Modal Sensory Compensation Effects of Ambient Scent on Food Purchases. Journal of Marketing Research, 56(1), 123-141. 
Jiménez-Marín, G.; Bellido-Pérez, E. y López-Cortés, A. Marketing sensorial: el concepto, sus técnicas y su aplicación en el punto de venta.

Braidot, N. (2014). Neuromanagement: Del Management al Neuromanagement. L'hospitalet de Llobregat: Granica.

Braidot, N. (2011). Neuromarketing en el punto de venta. El efecto de la música. Estrategia magazine, septiembre de 2011.

Bushdid, C.; Magnasco, M. O.; Vosshall, L. B.; y Keller, A. (2016). Humans Can Discriminate More than 1 Trillion Olfactory Stimuli. Science, 343, 1370-1372.

Castanyol i Casals, E. (2014). Marketing sensorial: comunicación a través de los sentidos. COMeIN - Revista de los estudios de ciencias de la información y comunicación, 38.

Cruz García, M. J. (2012): Ensayo sobre el neuromarketing. Veracruz: Instituto Tecnológico de Monterrey.

Damasio, A. R. (1994). El error de Descartes. Barcelona: Destino.

De Garcillán López-Rúa, M. (2015). Persuasión a través del marketing sensorial y experiencial. Opción: Revista de Ciencias Humanas y Sociales, No. Extra 2, 463-478.

Devans, N. A. (2005). El valor de la publicidad sensorial. Razón y palabra, 46(15), Recuperado de http://www.razonypalabra.org.mx/anteriores/n46/nalvarez.html

Díaz, J. (2012). Cómo atraer a los clientes a través de los cinco sentidos. Negocios $y$ emprendimiento. Ideas $y$ herramientas para emprender. Recuperado de http:// www.negociosyemprendimiento.org/2012/05/como-atraer-los-clientes-travesde-los.html

Ferrada, S. (2013). Cómo activan las marcas nuestro 'botón de compra'. El Huffington Post. Recuperado de http://www.huffingtonpost.es/santiago-ferrada-/marcas-boton-decompra_b_4217523.html

Ferrés, J. (1996). Televisión subliminal. Socialización mediante comunicaciones inadvertidas. Barcelona: Paidós.

Gómez Ramírez, C. (2012). La identidad olfativa: una estrategia invisible y silenciosa. Revista Virtual, 37, 156-179.

Gómez Suárez, M. y García Gumiel, C. (2012). Cómo desarrollar la atmósfera del establecimiento comercial. Distribución y consumo, 30-39. 
Jiménez-Marín, G.; Bellido-Pérez, E. y López-Cortés, A. Marketing sensorial: el concepto, sus técnicas y su aplicación en el punto de venta.

Guerra Serrano, A.; Gomes-Franco e Silva, F. (2017). El uso del neuromarketing y del marketing sensorial en los eventos un estudio de caso. Redmarka: revista académica de marketing aplicado, 18, 21-47.

Gulas, C. S. y Bloch, P. H. (1995). Right under our noses: ambient scent and consumer responses. Journal of Business and Psychology, 10(1), 87-98.

Gustems Carnicer, J. (2005). Escuchar los anuncios. Una aproximación al uso de la música y del sonido en la publicidad televisiva. Eufonía: Didáctica de la música, 34, 91-100.

Haase, J. \& Wiedmann, K. P. (2018). The sensory perception item set (SPI): An exploratory effort to develop a holistic scale for sensory marketing. Psychology \& Marketing, 1-13. DOI: 10.1002/mar.21130

Hernández Alarcón, E. (2005). Evaluación sensorial. Bogotá: Universidad Nacional Abierta y a Distancia.

Hulten, B. (2011). Sensory marketing: the multi-sensory brand-experience concept. European Business Review, 23(3), 256-273.

Jiménez-Marín, G. y Elías Zambrano, R. (2018). Marketing sensorial: merchandisig a través de las emociones para llegar al consumidor. Análisis de un caso. AdComunica. Revista Científica de Estrategias, Tendencias e Innovación en Comunicación, Vol. X., 235-253.

Jiménez-Marín, G. (Ed) (2017). La gestión professional del merchandising. Barcelona: UOC.

Jiménez-Marín, G. (2016). Merchandising \& Retail. Comunicación en el punto de venta. Sevilla: Advook.

Krishna, A. (2012). An integrative review of sensory marketing: Engaging the senses to affect perception, judgment and behavior. Journal of Consumer Psychology, 22, 332-351.

Krishna, A. (2010). Sensory marketing: Research on the sensuality of products. Nueva York: Routledge.

Krishna, A., Cian, L. \& Sokolova, T. (2016). The power of sensory marketing in advertising. Current Opinion in Psychology, 10, 142-147.

Leclerc, F., Schmitt, B. H. \& Dube, L. (1994). Foreign branding and its effects on product perceptions and attitudes. Journal of Marketing Research, 31(2), 263-270.

Vivat Academia. Revista de Comunicación. 15 septiembre 2019/15 diciembre 2019, nº 148, 121-147 
Jiménez-Marín, G.; Bellido-Pérez, E. y López-Cortés, A. Marketing sensorial: el concepto, sus técnicas y su aplicación en el punto de venta.

Lee, L., Frederick, S., \& Ariel, Y. D. (2006). Try it, you'll like it: The influence of expectation, consumption, and revelation on preferences for beer. Psychological Science, 17(12), 10541058.

Levin I. P., y Gaeth, G. J. (1988). How consumers are affected by the framing of attribute information before and after consuming the product. The Journal of Consumer Research, 15, 374-378.

Lindstrom, M. (2012). Buyology: verdades y mentiras sobre por qué compramos. Barcelona: Gestión 2000.

Llinás, R. R. (2003). El cerebro y el mito del yo: el papel de las neuronas en el pensamiento y el comportamiento humanos. Barcelona: Belacqua.

Lobato Gómez, F. (2005). Marketing en el punto de venta. Madrid: Thomson Paraninfo.

Manzano, R., Gavilan, D., Avello, M., Abril, C. y Serra, T. (2012). Marketing sensorial: comunicar con los sentidos en el punto de venta. Madrid: Pearson Educación.

Martí Parreño, J. (2012). Determinantes de la eficacia publicitaria actual: el Modelo AMBER (Atención-Motivación-Brand Engagement-Respuesta). Questiones Publicitarias, 17, 122138.

Monge Benito, S. y Fernández Guerra .V. (2011). Neuromarketing: Tecnologías, Mercado y Retos. Pensar la Publicidad, 5(2), 19-42.

Mukherjee, S. (2015). 'Brainfluence'-The Effectual Appeal to Customer Psyche. Advances in Economics and Business Management (AEBM), 2(13), 1291-1295.

Nenkov, G. Y., Morrin, M., Maille, V., Rank-Christman, T., y Lwin, M. O. (2018). Sense and sensibility: The impact of visual and auditory sensory input on marketplace morality. Journal of Business Research. DOI: 10.1016/j.jbusres.2018.07.047

Peck, J. y Childers, T. L. (2003). Individual differences in haptic information processing: 'the need for touch' scale. Journal of Consumer Research, 30(3), 430-442.

Peck, J., Barger V. A. \& Webb, A. (2013). In search of a surrogate for touch: the effect of haptic imagery on perceived ownership. Journal of Consumer Psychology, 23, 189-196.

Peck, J. \& Shu, S. B. (2009). The effect of mere touch on perceived ownership. J Consumer Research, 36, 434-447. 
Jiménez-Marín, G.; Bellido-Pérez, E. y López-Cortés, A. Marketing sensorial: el concepto, sus técnicas y su aplicación en el punto de venta.

Petit, O., Velasco, C. \& Spence, C. (2019). Digital Sensory Marketing: Integrating New Technologies Into Multisensory Online Experience. Journal of Interactive Marketing, 45, 4261.

Plassmann, H., Ramso, T. Z., \& Milosavljevic, M. (2012). Branding the brain: A critical review and outlook. Journal of Consumer Psychology, 22, 18-36.

Popai (2016). Informe Popai. Recuperado de http:// www.popaispain.org

Ramiro, P. (2017). La última campaña de la DGT te hace experimentar un accidente. El País. Recuperado de http://politica.elpais.com/politica/2017/04/27/actualidad/1493294517_368467.html

Ramos Serrano, M.; Delgado Brull, M. T. y Jiménez-Marín, G. (2007). Las nuevas estrategias de comunicación en el sector automovilístico: razón y emoción en la campaña de Audi. FISEC-Estrategias, año III, 6, 23-45. Recuperado de https://www.researchgate.net/publication/228810357_las_nuevas_estrategias_de_com unicacion_en_el_sector_automovilistico_razon_y_emocion_en_la_campana_de_audi

Roberts, K. (2005). Lovemarks. El futuro más allá de las marcas. Madrid: Empresa activa.

Sánchez Franco, M. J. (1999). Eficacia publicitaria. Madrid: McGraw-Hill.

Santos de Almeida, L. R. (2018). Neuromarketing: A nova ciencia do consumo. Revista de Administraçâo de Empresas, 58(4), 433-444.

Santos, F. (2013). Marketing sensorial y el imperio de los sentidos. Puro Marketing. Recuperado de http://www.puromarketing.com/44/18230/sensorial-imperosentidos.html

Schiffman, L. G. \& Kanuk; L. L. (2005). Comportamiento del consumidor. México: Prentice Hall.

Serrano Aban, N., \& De Balanzó Bono, C. (2010). Neuromarketing y Memoria: Implicaciones para la Comunicación Publicitaria. Pensar la publicidad, 6(2), 297-313.

Shu, S. B., \& Peck J. (2011). Psychological ownership and affective reaction: emotional attachment process variables and the endowment effect. Journal of Consumer Psychology, $21,439-452$.

Stanton, S. J., Sinnott-Armtrong, W. \& Huettel, S. A. (2017). Neuromarketing: Ethical Implications of its Use and Potential Misuse. Journal Bus Ethics, 144, 799-811. 
Jiménez-Marín, G.; Bellido-Pérez, E. y López-Cortés, A. Marketing sensorial: el concepto, sus técnicas y su aplicación en el punto de venta.

Toller, S. Van \& Dodd, G. H. (2013). Perfumery: The Psychology and Biology Fragrance. Nueva York: Springer Science \& Business Media.

Valenti, C. \& Riviere, J. (2008). The concept of Sensory Marketing. Högskolan i Halmstad: Marketing dissertation.

Vroom, P. (1999). La seducción secreta. Psicología del olfato. Barcelona: Tusquets.

Wansik, B., Park, S., Sonka, S. \& Morganosky, M. (2000). How soy labeling influences preference and taste. Management Review, 3, 85-94.

\section{AUTORAS}

\section{Gloria Jiménez-Marín}

Doctora por la Universidad de Sevilla y Profesora en el área de Publicidad y RR.PP. en la Facultad de Comunicación de la misma universidad. Licenciada en Periodismo y Licenciada en Publicidad y RR.PP., colabora, además, con la Universitat Oberta de Catalunya y es Máster en Diseño Publicitario. Tras su paso por la actividad profesional en agencias de publicidad y su labor docente en varias universidades españolas, su actividad investigadora se centra en el estudio de la relación distribución comercial comunicación a través del merchandising en los espacios comerciales así como en las relaciones entre la publicidad y las diferentes disciplinas artísticas.

gloria_jimenez@us.es

Orcid ID: https:/ / orcid.org/0000-0003-0252-3975

Google Scholar: https:// scholar.google.es/citations?user=pNLUjXUAAAAJ\&hl=es

Research ID: E-5845-2010

Scopus: https:// www.scopus.com/authid/detail.uri?authorId=55580687600

\section{Elena Bellido-Pérez}

ebellido@us.es

Orcid ID: https:// orcid.org/0000-0002-3107-5481

ResearchGate: https://www.researchgate.net/profile/Elena_Bellido-Perez

\section{Ángela López-Cortés}

Ángela López-Cortés es graduada en Publicidad y Relaciones Es beneficiaria de un contrato predoctoral o de Personal Investigador en Formación (PIF) en la Facultad de Comunicación de la Universidad de Sevilla. Graduada en Publicidad y Relaciones Públicas, donde obtuvo el Premio Extraordinario Fin de Estudios de la Universidad de Sevilla, y Máster en Comunicación y Cultura por la Universidad de Sevilla, actualmente Elena está desarrollando su tesis doctoral sobre arte y propaganda dentro del Doctorado Interuniversitario en Comunicación, por la Universidad de Cádiz, Huelva, Málaga y Sevilla. Realiza su actividad docente e investigadora dentro del Departamento de 
Jiménez-Marín, G.; Bellido-Pérez, E. y López-Cortés, A. Marketing sensorial: el concepto, sus técnicas y su aplicación en el punto de venta.

Comunicación Audiovisual y Publicidad, y en el seno del grupo de investigación IDECO. Públicas en el año 2017 en la Facultad de Comunicación de la Universidad de Sevilla. En 2018 realizó un Máster en Comunicación y Cultura en esta misma universidad. Posee formación complementaria como experta en protocolo militar y organización de eventos. angelalopezc@us.es 